j. port. sci. res.
ISSN: $2616-7441$ (Online)
ISSN: 2616-6232 (Print)
ISSN: 2616-7220 (USB)

\title{
Effect of piezoelectric material on vibration of vessel of marine transportation
}

\author{
S. K. Mohammed \\ College of Engineering, Uruk University. Baghdad. Iraq \\ Salsabil2alanber@gmail.com
}

\begin{abstract}
The drive arrangement of normal marine vessels involves a propeller coupled to a progression of shafts and diary orientation which is at last associated with a push bearing which sends the propeller pivotal power into the frame of the boat through a push block coupled to the mass head. For effortlessness, the framework is improved to a propeller, a solitary shaft, diary bearing and a push bearing. As the vessel travels through the sea, a wake is produced. This non-uniform wake field is the inflow to the propeller. The power produced by the propeller is additionally non-uniform. The impetus arrangement of a marine vessel is the principle supporter of commotion emanated from the vessel. It is along these lines important to diminish the commotion sent from the propeller into the sea and consequently the vibration that is created by the propeller that is thus communicated into the body. The inspiration for lessening commotion and vibration remembers diminishing mechanical wear for segments, expanding secrecy limit of military vessels, improving traveler and group solace, and decreasing the effect on marine conditions. The point of this theory is to dissect the shaky power created by the propeller, the power transmission ways, and techniques to quantify the power transmission through the push bearing continuously.
\end{abstract}

Crossref

10.36371/port.2021.2.1

Keywords: piezoelectric; ovibration; vessel; mechanical; noise

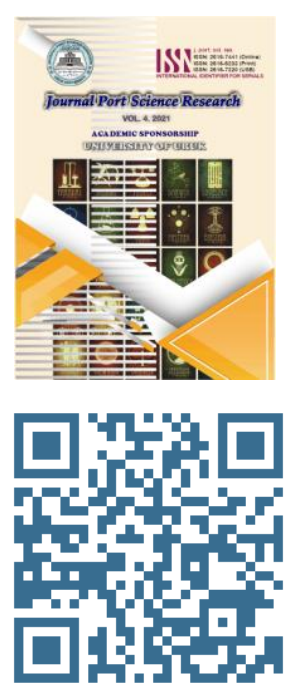

\section{INTRODUCTION}

This commotion is produced by the vessel's locally available frameworks, for example, its impetus framework, motor and inboard apparatus. Lessening the commotion emanated out of these vessels has a plenty of points of interest: the biggest being the increment in their secrecy limit. Diminishing the commotion created additionally brings about diminished team weakness, expanded traveler comfort just as decreased mechanical wear[1]. The propeller is the biggest single supporter of the general commotion created by the framework. The clamor can be sent by means of two ways:

1) the immediate transmission of the hydrodynamic commotion created by the propeller;

2) the roundabout way where the commotion is being communicated through the propeller to the shaft, at that point through the push bearing lastly into the frame and the sea. [2]

The birthplace of the propeller commotion comes from two unmistakable sources. The first happens at low propeller speeds where the propeller inflow is influenced by the procedure body wake which brings about lopsided stacking produced across the propeller face. These lopsided burdens bring about vibrations being sent down the shaft, through the push bearing and into the body bringing about emanated commotion. This commotion has normal apparent attributes that happen at the edge passing recurrence (BPF) and its music. The second birthplace of the commotion happens at cruising propeller tip speeds where the cooperation of the propeller and water brings about fierce ingestion clamor that can be straightforwardly transmitted out just as being coupled into the body commotion. This commotion anyway is not the same as the first since it brings about broadband clamor excitation up to $10 \mathrm{kHz}$. It very well may be limited through improved propeller plan, or diminishing the meaning of the frame wake . Recognizing the transmission ways of the marine impetus frameworks and detaching these ways may lessen the absolute acoustical mark of the vessel[3] .

Push course permit rotational movement under enormous pivotal power while producing negligible grinding. The shifted cushion push bearing, created by Kingsbury [1950b] , is found in most marine impetus frameworks. In its most straightforward structure, it involves a discrete number of cushions at a fixed point similarly dispersed. More complex plans license the cushions to consequently change their point to suit the working pressing factors. Despite the bearing sort, liquid filled direction all depend on the pivotal burden being upheld by a flimsy layer of oil. The push bearing's firmness and damping attributes impact the commotion produced by the propeller that is vibrated to the frame. The frame's vibration qualities are additionally impacted by its rib

Mohammed, S., Effect of piezoelectric material on vibration of vessel of marine transportation. Journal port Science Research, 4(2). pp 46-52. https://doi.org/10.36371/port.2021.1.8 
structure as the framework is coupled. Being able to precisely demonstrate pushed bearing execution would empower enhancement strategies to be created to diminish vibration transmission through the marine impetus framework[4].

\section{LITERATURE REVIEW}

\subsection{PVDF sensors}

For functional applications, Polyvinylidene Fluoride (PVDF) sensors have numerous aggregate focal points, for example, wide scope of recurrence reactions, vibration location or effect detecting, and acoustic emanation discovery. PVDF is a semi-glasslike polymer that can emerge in a few translucent states. One of these states is ferroelectric and is the explanation behind its characteristic piezoelectric material property. PVDF piezoelectric film sensors being used today present another estimating method that could tackle power part issues. One such method includes utilizing a variety of 4 PVDF films divided evenly about the hub of evenness. There are a wide range of sorts of PVDF films with different sensitivities accessible on the lookout, anyway Measurement Specialties supply the most usually utilized movies[3] .

PVDF experiences anisotropy meaning it has various reactions in various ways. These various reactions are presented when the film is exposed to a uniaxial stacking while at the same time being heat treated between 60 to $80^{\circ} \mathrm{C}$ .. Anodes are set on the two sides of the PVDF film, and it is thermally enraptured at $100^{\circ} \mathrm{C}$ in an electric field of 500 to $800 \mathrm{kV}$. The film is then cooled in the electric field to "freeze" the polarization.[5]

The alignment of these PVDF films has demonstrated troublesome. This is generally because of the large number of alignment strategies that have been referenced in writing, and their anisotropic nature. An illustration of this is introduced by Soyama et al. [1998] who utilized PVDF to quantify the effect of cavitation, however,the sensors required alignment for such a quick stacking. To defeat this, the snap of a graphite pencil,as the length of the effect coordinates that of cavitation bubbles, was utilized to energize the PVDF which was upheld on a knifes-edge. It was likewise clarified that the yield of the PVDF films is relative to the time the power is applied. This anyway isn't relevant here as consistent unique stacking will be likely[6] .

Another adjustment method was utilized by a few investigations who utilized a pendulum-type ball sway strategy where the anticipated idea of a pendulum strike was utilized to energize the PVDF film. The applied burden can then determined and contrasted with the yield of the PVDF to give a type of affectability of the PVDF in that specific application. This procedure seems helpful as it is direct to compute the energy applied by a strike of a pendulum. [1]

\subsection{Vibration control}

Outside of control methods encompassing engine, shaft and bearing misalignment a great deal of examination has been done on assistant gadgets that can improve the current framework. A wide range of strategies have been proposed including inactive, semi-dynamic and dynamic procedures . These investigations had restricted achievement in vibration constriction of impetus frameworks and could profit by a more precise model of propeller prompted powers[7] .

Rigby [1948] proposed that expanding the quantity of sharp edges of the propeller could lessen the abundancy of the framework vibration. Nonetheless, expanding the quantity of cutting edges prompts a deficiency of productivity and expanded danger of cavitation. The paper likewise thought about framework vibration for various push block positions and explore the impacts of shaft adaptability. Goodwin [1960] traces the benefits of assistant frameworks, explicitly a reverberation transformer (RC) which plays out the undertaking of a water driven unique vibration safeguard, as it kills the strange situating of push obstructs or changing the quantity of sharp edges which doesn't generally limit inordinate vibration. The reverberation transformer was found to sufficiently dispense with hub vibration of the impetus framework under full conditions. a few scientists utilized the limited component/limit component model of a lowered submarine to notice the effect of a $\mathrm{RC}$ on the framework vibration[8]. The RC was discovered to be restricted over specific frequencies and add to the sound field that energizes the body. a few examinations referenced that aloof control is just viable at higher frequencies and dynamic control will in general be costly and ineffectual. As traveler distress and most serious harm to send structures are credited to low recurrence vibration their examination researches elective control techniques, for example, underlying alteration[9] .

Different investigations have explored vibration control techniques including dynamic vibration safeguards, electromagnetic dampers and reverberation transformers with restricted achievement . Giving a superior comprehension of the basic boundaries that add to propeller actuated vibration is attractive. This arrangement would give better bearing to future control instruments and key capacities expected to successfully diminish the vibration.

\section{METHODOLOGY}

\subsection{Energy Flowing into the Piezoelectric System}

The piezoelectric transducer goes through pressure applied by the cantilever shaft. A piece of the dynamic energy, EKPZ, goes into the piezoelectric transducer and, because of its

Mohammed, S., Effect of piezoelectric material on vibration of vessel of marine transportation. Journal port Science Research, 4(2). 46-52. 
electromechanical coupling attributes, is changed over into electrical energy, EHPZ. [10]

\subsubsection{Open Circuit conditions}

Under open circuit condition, for example the piezoelectric transducer isn't associated with a heap, this electrical energy, EHPZ, is incidentally put away in the piezoelectric capacitance . At that point because of the auxiliary piezoelectric impact this electric energy, EHPZR, gets back to the mechanical arrangement of the Hybrid $\mathrm{VEH}$, for example into the cantilever bar underlying energy, EHS. [11]

\subsubsection{Load Conditions}

On the off chance that there is a heap associated with the piezoelectric transducer the changed over electrical energy, EHPZ, has another dissemination.. A part of this energy, ETH2, because of the electrical obstruction of the piezoelectric material is definitely lost as warmth (hued in red). Another piece of this electrical energy, EHPZ, is conveyed to the heap (shaded in blue) or put away in an energy stockpiling gadget. The measure of this energy is considered as collected and is the last objective of the energy change measure[12]. In the event that the electronic circuit between the piezoelectric transducer and the heap doesn't permit whole conveyance of the electric energy to the heap, a piece of this energy, EHPZR, returns once again into mechanical arrangement of the Hybrid VEH, for example into the cantilever bar underlying energy, EHS, as in the open circuit conditions.

\subsection{Energy Flowing into the Electromagnetic System}

There is a nearby similitude between energy stream in piezoelectric and electromagnetic frameworks. Nonetheless, the energy changing over component and the period of transformation for the piezoelectric and electromagnetic transducers are unique. Because of dormancy, the tip mass uprooting slacks the removal of the lodging. The piezoelectric energy change is reliant on relocation extent of the point, some time the electromagnetic energy transformation relies upon the speed of the tip mass[13]. Consequently, the electromagnetic energy transformation slacks the piezoelectric energy change by a specific point. Notwithstanding, on account of cantilever pillar Hybrid VEH the two transducers have similar phases of energy stream: transformation (beginning of the energy stream), cycling of electrical energy in circle, scattering, and moving the energy to a heap . [14]

A piece of the put away energy in the cantilever shaft primary energy, EHS, changes into active energy, EKEM, of the moving tip mass. This motor energy goes into the electromagnetic transducer and because of its electromechanical coupling attributes is changed over into electrical energy, EHEM.

\subsubsection{Open Circuit conditions}

Under open circuit condition, for example the electromagnetic transducer isn't associated with a heap, this electrical energy, EHPZ, is briefly put away in the electromagnetic field. At that point because of Lenz' law this electric energy, EHEM, gets back to the mechanical arrangement of the Hybrid $\mathrm{VEH}$, for example into the cantilever bar underlying energy, EHS. The actuated potential in the loop makes electrical flow which delivers an attractive field that will in general contradict the change in attractive motion that initiates this flow. In this manner, there is an attractive field inverse to the attractive field of the lasting magnet and the energy of the contradicting attractive field returns energy, EHEMR back to the cantilever shaft. [15]

\subsubsection{Load Conditions}

On the off chance that there is a heap associated with the electromagnetic transducer the changed over electrical energy, EHEM, has another circulation. Because of Lenz's Law this electrical energy begins to course in the energy circle . A bit of this energy, ETH3, because of the electrical obstruction of the loop material is definitely lost as warmth (hued in red). [16]Another piece of this electrical energy, EHEM, is conveyed to the heap (shaded in blue) or put away in energy stockpiling gadget. The measure of this energy is considered as reaped and is the last objective of the energy change measure. In the event that the electronic circuit between the piezoelectric transducer and the heap doesn't permit whole conveyance of the electric energy to the heap, a piece of this energy, EHEMR returns once again into the mechanical arrangement of the Hybrid VEH, for example into the cantilever bar primary energy, EHS, as in open circuit conditions.

\subsection{Harvesting of Energy from Piezoelectric and Electromagnetic Systems}

Once went into the electrical piezoelectric and electromagnetic frameworks, electric energy can be gathered, for example used to control a heap or put away in an energy stockpiling gadget. Definitely, a piece of this energy (the amount of ETH22 and ETH33) is scattered due to the nonideal attributes of electrical segments of the interface circuits. The extension rectifier for instance burns-through force because of the diodes forward voltage drop as does the voltage multiplier as well. [7]

The separated electrical energy from the piezoelectric transducer, EPZEL, and from the electromagnetic transducer, EEMEL, is conveyed to the heap under various conditions. 
Normally, a piezoelectric transducer delivers a lot higher yield voltage than an electromagnetic transducer. Hence, various burdens are used for gathering the electric energy on account of the half breed reaper as introduced in the writing up until this poin.

In the event that the electrical energy from the two transducers is conveyed under various conditions into a typical burden one of them could burn-through the energy created by the other. To maintain a strategic distance from such one circumstance an electronic interface for coordinating yield impedances of the transducers and changing the yield voltages is proposed in this exposition.

\subsubsection{Energy Flowing into and Returning to the Mechanical System}

the portions in white shading present the flowing energy as mechanical energy, EHMC, for the mechanical framework and electrical energy, EHPZC, and EHEMC for the electrical frameworks. Most of all entering in the Hybrid VEH energy stays in the mechanical framework. A bit of the energy continues to cycle in the mechanical and electrical frameworks of the piezoelectric and electromagnetic transducers. As referenced above, if the electronic circuit doesn't permit whole usage of this energy by the heap the bit of this energy, EKPZR and EKEMR, gets back to the mechanical arrangement of the Hybrid VEH. [3]

In the event that the absolute mechanical impedance of the half breed piezoelectric and electromagnetic gatherer doesn't coordinate the vibration source impedance, a piece of the flowing energy in the mechanical framework energy gets back to the vibration source as motor energy, EKTR.

\subsection{Efficiency Evaluation of the Hybrid VEH}

A Sankey energy stream chart presents the 10,000 foot view of the energy dispersion inside the Hybrid VEH. Area of predominant streams and correlation with the general energy stream is conceivable for this situation. [13]

In a few investigations are given three portraying boundaries: gathering factor, $\eta \mathrm{h}$, dispersal factor $\eta \mathrm{d}$, and misfortune factor, $\eta 1$, which on account of the Hybrid VEH are communicated by (1), (2), and (3). The cycling energy, EHMC, is assessed per one cycle thus there is an augmentation by $2 \pi$ to get the complete cycling energy for this cycle.

$$
\begin{aligned}
& n_{h}=\frac{E \mathrm{C}}{2 \pi \mathrm{E}_{\mathrm{HMC}}}=\frac{E_{P E Z L}+E_{E M E L}}{2 \pi \mathrm{E}_{\mathrm{HMC}}} \\
& n_{d}=\frac{\mathrm{Eh}}{2 \pi \mathrm{E}_{\mathrm{HMC}}}=\frac{\mathrm{E}_{\mathrm{TH} 2}+\mathrm{E}_{\mathrm{TH} 3}+\mathrm{E}_{\mathrm{TH} 22}+\mathrm{E}_{\mathrm{TH} 33}}{2 \pi \mathrm{E}_{\mathrm{HMC}}}
\end{aligned}
$$

$$
\eta_{I}=\eta_{h}+\eta_{d}=\frac{E_{P Z E L}+E_{E M E L}+E_{T H 2}+E_{T H 3}+E_{T H 22}+E_{T H 33}}{2 \pi E_{H M d}}
$$

Where.

Eh is harvested by piezoelectric and electromagnetic transducers energy in one cycle, [Wh];

$\mathrm{Ed}$ is dissipated energy in one cycle in piezoelectric and electromagnetic systems, [Wh]; EPZEL is harvested by piezoelectric transducers electric energy, [Wh];

EEMEL is harvested by electromagnetic transducers electric energy, [Wh];

ETH2 is harvested by piezoelectric and electromagnetic transducers energy, [Wh]; ETH3 is harvested by piezoelectric and electromagnetic transducers energy, [Wh];

ETH22 is dissipated by piezoelectric transducers energy due to non-ideal characteristics of electrical components, [Wh];

ETH33 is dissipated by electromagnetic transducers energy due to non-ideal characteristics of electrical components, [Wh];

EHMC is the cycling in the mechanical loop energy, [Wh].

\section{RESULTS}

As can be seen from the qualities in Table 1, the sensitivities on the whole three bearings (1,2 and 3$)$ have been significantly changed by implanting the sensor in different materials. The 3 way, the sensor's reaction has been decreased by around a factor of 10 for every one of the three squares $(0.8,1.5$ and $1.1 \mathrm{mV} / \mathrm{N}$ for the Aluminum Stratasys and ABS plastic squares separately) when contrasted with the uncovered film $(14 \mathrm{mV} / \mathrm{N})$. This decrease is normal since the applied power will be lessened as the power goes through the square into the PVDF film

The 1 way, the sensitivities all have negative qualities showing that the 31 and 32 voltage segments are being energized. For the aluminum block, these voltages are more modest than the two plastic square's reaction. It is fascinating to take note of the sensitivities taking all things together three bearings are tantamount in size. This is amazing since the $\mathrm{g} 31$ piezoelectric consistent is by and large comprehended to be zero or, best case scenario, one 10th of $\mathrm{g} 32$. S the yield in the 1 and 2 ways because of powers applied in these ways is zero, the negative reactions noticed should be because of the development of compressional strain in the comparing bearing. The bizarrely high estimations of the sensitivities in the ABS plastic square are no doubt because of the nonhomogeneous nature of the material. Regardless of the affectability esteems follow no unmistakable pattern, obviously applying a straightforward symmetrical power to 
the outside of the square can bring about a complex actuated strain field created in the PVDF. This has critical ramifications on the voltage yield of this sensor which is a superposition of the voltage in the three ways given by Equation 4. Therefore, it was chosen to demonstrate the PVDF and square design utilizing FEM .

Output as a component of point

For reasonable applications, the interior powers because of outside excitation detected by the film won't really be opposite to those of the three piezoelectric tomahawks. These powers may change in greatness as well as in bearing. To explore these impacts, a similar Stratasys block was utilized to gauge the affectability as a component of point toward every path independently. To do this, it was first important to change the tip of the effect sledge with the end goal that it was conceivable to apply the power at a limited point. This was finished by supplanting the current Aluminum tip with a little steel tip that had been machined to a sharp point. To precisely control the course of the information power, a little plastic support framework was built utilizing a 3D printer. The effect hammer was gotten inside the support and situated at different points over each surface of the square. The point was estimated utilizing an attractively mounted inclinometer that was eliminated preceding estimations.

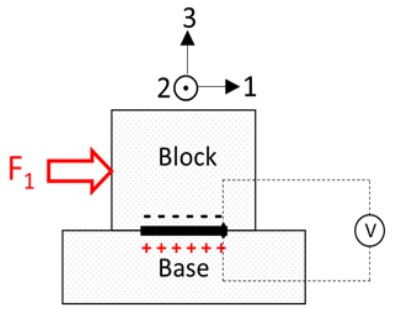

(a)

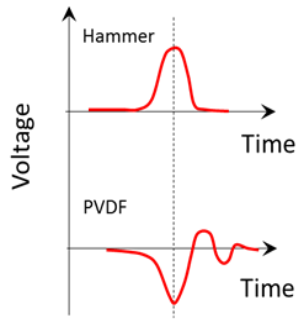

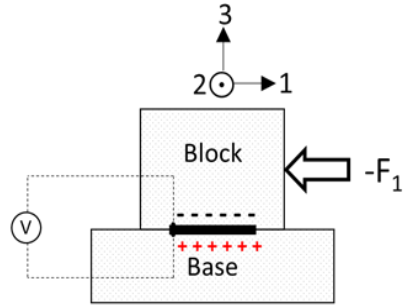

(b)

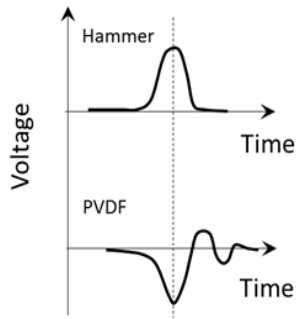

Figure 1: Observed output voltage for a force applied parallel in the positive and negative 1 direction of the PVDF film.

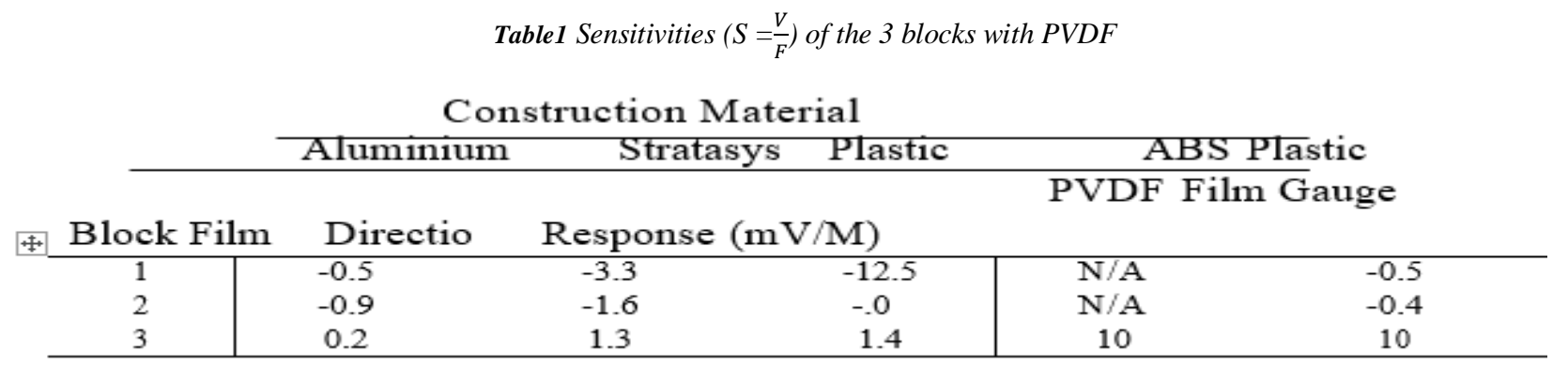

$T$

able 2: Coefficient of determination of each of the directions in each structure

Construction Material

Aluminium Plastic

3D Printed Plastic

\begin{tabular}{c|ccc}
\hline Direction & \multicolumn{3}{c}{ Coefficient of Determination } \\
\hline 1 & 0.9270 & 0.9918 & 0.8074 \\
2 & 0.8416 & 0.9723 & 0.8010 \\
3 & 0.8956 & 0.9345 & 0.8019 \\
\hline
\end{tabular}

\section{Conclusion}

PVDF has a straight affectability bend for every one of the detecting bearings 1,2 and 3 . These were estimated when the PVDF film was inserted inside a straightforward square math. Three indistinguishable square constructions were produced using three unique materials: aluminum, 3D printed $\mathrm{ABS}$ plastic and 3D printed Stratasys 720 Full fix ink. These immediate sensitivities were estimated when hit with an effect hammer. The voltage of the sensors was contrasted with that of the power applied by the mallet. It was discovered that the power transmission into the PVDF film was diverse for every one of the designs. It was additionally discovered that the general change between the delicate headings was the equivalent for every one of the materials which recommends the thing that matters was the material properties. 


\section{REFERENCES}

[1] Kelley, C. R., Lopp, G. K., \& Kauffman, J. L. (2021). Optimizing Piezoelectric Material Location and Size for MultipleMode Vibration Reduction of Turbomachinery Blades. Journal of Vibration and Acoustics, Transactions of the ASME, 143(2). https://doi.org/10.1115/1.4048263 Ding, D., Surface Characterization of Piezoelectric Sensor Materials for Potential Use in Reactor Vessel Sensors. 2019.

[2] Mohammadi, M., Bamdad, M., Alambeigi, K., Dimitri, R., \& Tornabene, F. (2019). Electro-elastic response of cylindrical sandwich pressure vessels with porous core and piezoelectric face-sheets. Composite Structures, 225. https://doi.org/10.1016/j.compstruct.2019.111119

[3] Meskini, M., \& Ghasemi, A. R. (2021). Electro-magnetic potential effects on free vibration of rotating circular cylindrical shells of functionally graded materials with laminated composite core and piezo electro-magnetic two face sheets. Journal of Sandwich Structures and Materials, 23(7), 2772-2797. https://doi.org/10.1177/1099636220909751

[4] Pan, L., Sun, S., Chen, Y., Wang, P., Wang, J., Zhang, X., ... Wang, Z. L. (2020, April 1). Advances in PiezoPhototronic Effect Enhanced Photocatalysis and Photoelectrocatalysis. Advanced Energy Materials. Wiley-VCH Verlag. https://doi.org/10.1002/aenm.202000214

[5] Mohammadi, M., Arefi, M., \& Ahmadi, S. A. (2020). Two-dimensional electro-elastic analysis of FG-CNTRC cylindrical laminated pressure vessels with piezoelectric layers based on third-order shear deformation theory. Journal of Pressure Vessel Technology, Transactions of the ASME, 142(2). https://doi.org/10.1115/1.4043842

[6] Rocha, R. T., Tusset, A. M., Ribeiro, M. A., Lenz, W. B., Haura Junior, R., Jarzebowska, E., \& Balthazar, J. M. (2020). On the Positioning of a Piezoelectric Material in the Energy Harvesting from a Nonideally Excited Portal Frame. Journal of Computational and Nonlinear Dynamics, 15(12). https://doi.org/10.1115/1.4048024

[7] Li, S., Liu, S., \& Yang, L. (2020). Active Control of Vibration and Noise of Energy Equipment. In IOP Conference Series: Earth and Environmental Science (Vol. 446). Institute of Physics Publishing. https://doi.org/10.1088/1755$\underline{1315 / 446 / 2 / 022076}$

[8] Sharma, P. (2021). Vibration analysis of FGPM beam: A review. In Materials Today: Proceedings (Vol. 44, pp. 13841390). Elsevier Ltd. https://doi.org/10.1016/j.matpr.2020.11.621

[9] Li, C., Liu, J. J., Cheng, M., \& Fan, X. L. (2017). Nonlocal vibrations and stabilities in parametric resonance of axially moving viscoelastic piezoelectric nanoplate subjected to thermo-electro-mechanical forces. Composites Part B: Engineering, 116, 153-169. https://doi.org/10.1016/j.compositesb.2017.01.071

[10] ugustine, R., Dan, P., Sosnik, A., Kalarikkal, N., Tran, N., Vincent, B., .. Rouxel, D. (2017). Electrospun poly(vinylidene fluoride-trifluoroethylene)/zinc oxide nanocomposite tissue engineering scaffolds with enhanced cell adhesion and blood vessel formation. Nano Research, 10(10), 3358-3376. https://doi.org/10.1007/s12274-017-1549-8

[11] Mir, O., Shakouri, M., \& Ashory, M. R. (2020). Gas pressure and density effects on vibration of cylindrical pressure vessels: analytical, numerical and experimental analysis. SN Applied Sciences, 2(1). https://doi.org/10.1007/s42452$\underline{019-1916-\mathrm{Z}}$

[12] Deng, L., Fang, Y., Wang, D., \& Wen, Z. (2018). A MEMS based piezoelectric vibration energy harvester for fault monitoring system. Microsystem Technologies, 24(9), 3637-3644. https://doi.org/10.1007/s00542-018-3784-7

[13] R. Bahaadini, M. Hosseini, M. A. Paparisabet. (2020). Vibration Analysis of Vessels Conveying Blood Flow Embedded in Viscous Fluid. Journal of Solid Mechanics, 12(4), 814-828. https://dorl.net/dor/20.1001.1.20083505.2020.12.4.7.9.

[14]Li, J., Dong, X., Zhang, G., Guo, Z., Zhang, G., \& Shi, C. (2021). An enhanced hemostatic ultrasonic scalpel based on the longitudinal-torsional vibration mode. IEEE Access, 9, $10951-10961$. https://doi.org/10.1109/ACCESS.2021.3050186 
[15]Qian, W., Zhao, K., Zhang, D., Bowen, C. R., Wang, Y., \& Yang, Y. (2019). Piezoelectric Material-Polymer Composite Porous Foam for Efficient Dye Degradation via the Piezo-Catalytic Effect.ACS Applied Materials and Interfaces, 11(31), 27862-27869. https://doi.org/10.1021/acsami.9b07857 